\title{
Distributed Control and Management Framework for Mobile and Multi-access Communication
}

\author{
Jukka Mäkelä and Markus Luoto \\ VTT Technical Research Centre of Finland \\ jukka.makela@vtt.fi, markus.luoto@vtt.fi
}

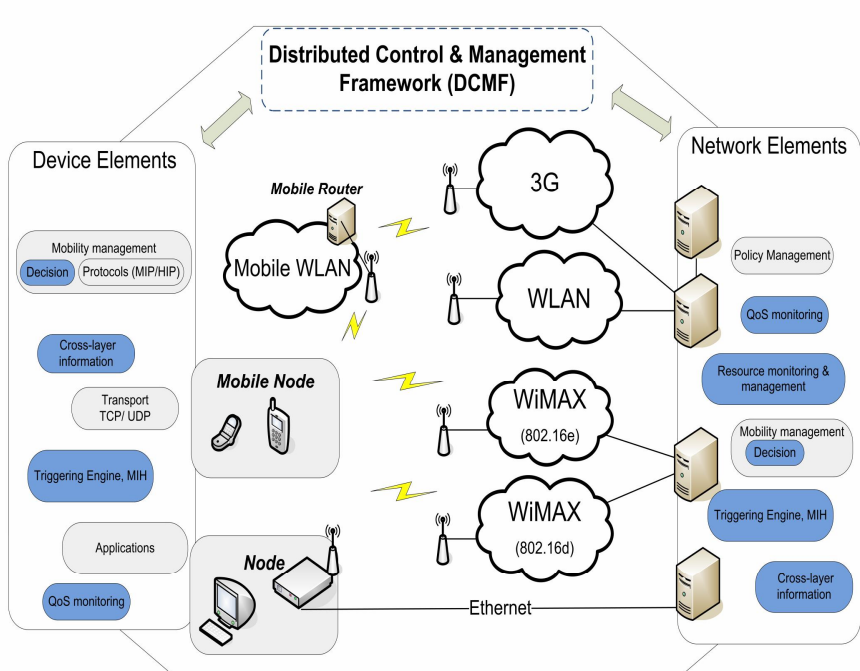

Figure 1. Elements of Distributed Control and Management system

\begin{abstract}
Concept
To support roaming, access control, and different optimizations in the network architecture, a new distributed control and management framework, shown in Figure 1, is required. The basis of this framework is a unified signalling architecture that is capable of serving different entities, located either in the network or in the terminals controlled by different players (terminals by the user, network by the service provider). The network side elements that would benefit from such a signalling framework include, for example, policy management, network/operator aided mobility management, and resource management. On the terminal side, mechanisms such as mobility management or transport protocol optimization solutions need access to extensive amount of information related to network access characteristics and roaming.

The information that is made available through the signalling system, can be used for optimizing network and terminal side operations in terms of, for example, applications' Quality of Service (QoS) requirements, energy efficiency, security, and network load balancing. In order to obtain relevant information for the decisionmaking process, we need to rely on, for example, operator policies, QoS measurements, and various cross-layer events (e.g. those defined IEEE 802.21).
\end{abstract}

\section{Prototype}

-Event based controlling framework

- Mobility triggering engine (TRG)

- Handover controlling based on various event input

- Signal strength, QoS, Network (congestion, user and application preferences

- Controlled IP-stream dissemination

- Session mobility between devices

- Network mobility with mobile router

- Access network information service

- Mobile IP and Host Identity Protocol

- Heterogeneous environment

- WiMAX 802.16 d/e, 3G/HSPA, WLAN

- IPv4/IPv6

- Linux, BSD, Windows, MAC OS, Maemo Linux

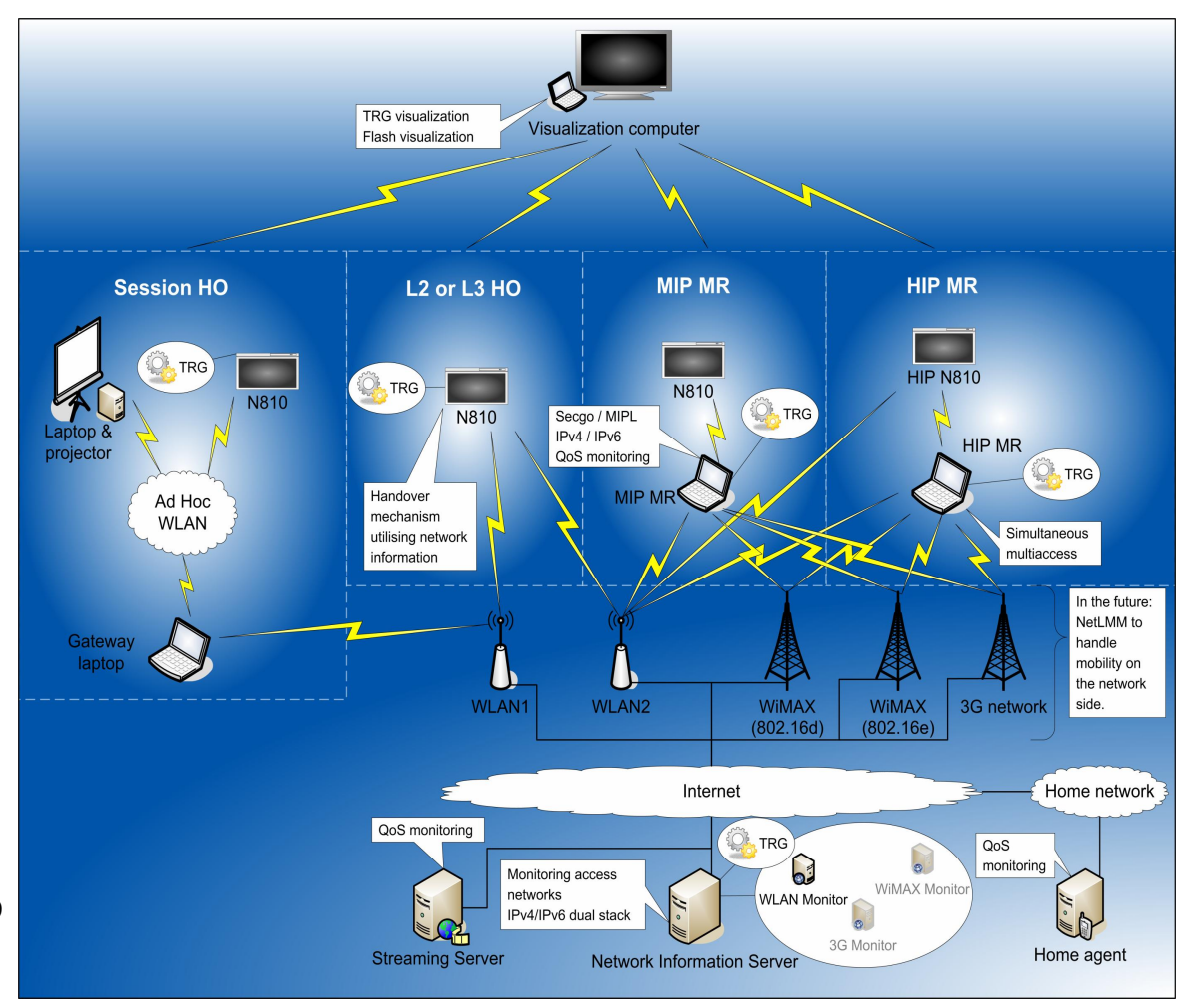

Figure 2. Prototype realization 